\title{
Lectures romantiques de la Passion du Christ et héritage des Lumières
}

\section{Paola Cattani}

\section{(2) OpenEdition}

Journals

\section{Édition électronique}

URL : http://journals.openedition.org/rief/246

DOI : $10.4000 /$ rief.246

ISSN : 2240-7456

\section{Éditeur}

Seminario di filologia francese

\section{Référence électronique}

Paola Cattani, «Lectures romantiques de la Passion du Christ et héritage des Lumières », Revue italienne d'études françaises [En ligne], 3 | 2013, mis en ligne le 15 décembre 2013, consulté le 03 mai 2019. URL : http://journals.openedition.org/rief/246 ; DOI : 10.4000/rief.246

Ce document a été généré automatiquement le 3 mai 2019.

\section{(c) (i) (9)}

Les contenus de la RIEF sont mis à disposition selon les termes de la Licence Creative Commons Attribution - Pas d'Utilisation Commerciale - Pas de Modification 4.0 International. 


\title{
Lectures romantiques de la Passion du Christ et héritage des Lumières
}

\author{
Paola Cattani
}

1 Jean Fabre, dans son Lumières et romantisme publié pour la première fois en 1963, mettait en garde contre le danger de représenter les XVIII ${ }^{e}$ et XIX ${ }^{e}$ siècles comme discontinus ; tentation qui menaçait à ses yeux en particulier les travaux de Paul Hazard ${ }^{1}$. Les périodisations critiques ont été, depuis lors, largement remises en question dans ce qu'elles ont d'artificiel et de rigide; et pourtant certaines associations demeurent, cristallisées dans notre mémoire collective du fait de leur longue persistance dans l'histoire des idées. L'une d'entre elles est l'opposition entre l'« écraser l'infâme » des Lumières et la religiosité romantique (au sens de la dévotion, mais aussi plus généralement de l'inquiétude spirituelle). Cette opposition a en effet connu une longue fortune dans l'imaginaire collectif notamment pour le rôle qu'elle a acquis auprès des écrivains "antimodernes", qui ont insisté sur le conflit entre identité romantique et raison des Lumières avec ses corollaires en matière de politique et de religion (une lecture qui au début du XXe siècle est mise en avant par exemple entre autres par Barrès et Maurras). Nous nous proposons de revenir ici sur cette opposition, afin de l'évaluer à partir d'une question spécifique : les lectures du thème de la Passion du Christ. C'est un auteur $\mathrm{du} \mathrm{XX}^{\mathrm{e}}$ siècle qui a attiré notre attention sur ce thème, Charles Péguy. Dans son dialogue inachevé Clio, Péguy propose une lecture particulière de l'épisode évangélique du Gethsémani : il présente le Christ comme faible et apeuré face à la mort qui va le saisir, à l'intérieur d'un discours visant à la célébration de la durée bergsonienne, de la sensibilité contre la raison, et du corps contre l'esprit. C'est sur les traces des sources de cette représentation du Christ, que nous sommes parvenue à explorer le tournant entre $\mathrm{XVIII}^{\mathrm{e}}$ et XIX ${ }^{\mathrm{e}}$ siècles : dans ce qui suit, nous tenterons de cerner le traitement du thème de la Passion à cette époque, à partir notamment de l'œuvre de Vigny, mais en élargissant le discours également à d'autres auteurs romantiques.

2 L'épisode évangélique du Christ qui invoque le père dans le jardin du Gethsémani en déclarant que son âme est «triste jusqu'à la mort » (Matthieu, XXVI, 36-46) et en implorant que le calice « passe loin » de lui (un passage qui depuis toujours a fait l'objet 
de controverses interprétatives, notamment à l'époque humaniste) ${ }^{2}$, retient l'attention de nombreux romantiques, qui presque tous transforment la prière de Jésus en un cri de désespoir de l'homme face à l'absence de Dieu. Le poème de Vigny Le Mont des Oliviers, de 1843, présente un Christ abandonné par ses compagnons, mais aussi par Dieu: «Puis regarde le ciel en appelant : "Mon Père !" / - Mais le ciel reste noir, et Dieu ne répond pas »3. Jésus réagit à ce silence d'abord avec stupeur («Il se lève étonné, marche encore à grands pas»), ensuite avec terreur ( Il recule, il descend, il crie avec effroi»), et finalement avec amertume, parvenant à une conclusion humaine et rationnelle qui exclut le divin ( «Il eut froid. Vainement il appela trois fois : / MON PÈre ! - Le vent seul répondit à sa voix. / Il tomba sur le sable assis et, dans sa peine, / Eut sur le monde et l'homme une pensée humaine »). La strophe finale, écrite en 1863, une vingtaine d'années après la première publication du poème, invite donc à dédaigner Dieu :

S'il est vrai qu'au Jardin sacré des Écritures,

Le fils de l'Homme ait dit ce qu'on voit rapporté ;

Muet, aveugle et sourd au cri des Créatures,

Si le Ciel nous laissa comme un monde avorté,

Le Juste opposera le dédain à l'absence

Et ne répondra plus que par un froid Silence

Au Silence éternel de la Divinité. ${ }^{4}$

3 De même, Nerval dans son Christ aux Oliviers, de 1844, transforme la douleur du Christ en un cri désespéré : «Et se prit à crier : “Non, Dieu n'existe pas !" » : la « nouvelle » n'est plus bonne, mais tragique :

Ils dormaient. «Mes amis, savez-vous la nouvelle?

J'ai touché de mon front à la voûte éternelle ;

Je suis sanglant, brisé, souffrant pour bien des jours !

Frères, je vous trompais : Abîme ! abîme ! abîme !

Le dieu manque à l'autel, où je suis la victime...

Dieu n'est pas! Dieu n'est plus ! » Mais ils dormaient toujours ${ }^{5}$

4 Et encore, Lamartine insère dans son Voyage en Orient le poème célèbre Gethsémani ou la mort de Julia, écrit après la mort de sa fille en 1832 : ici le mont des Oliviers devient le théâtre d'un rêve tragique annonçant la mort de la jeune fille. Le poète, qui se présente en "homme de désespoir » voué à l'agonie, élit comme refuge ce jardin de mort et de souffrance, en le préférant aux lieux saints des miracles de Jésus :

Conduisez-moi, mon père, à la place où l'on pleure !

À ce jardin funèbre où l'homme de salut,

Abandonné du père, et des hommes, voulut

Suer le sang et l'eau qu'on sue avant qu'on meure ;

Laissez-moi seul, allez, j'y veux sentir aussi

Ce qu'il tient de douleur dans une heure infinie.

Homme de désespoir, mon culte est l'agonie,

Mon autel à moi, c'est ici ! ${ }^{6}$

Le Christ qui prie et sue sang et eau devient pour Lamartine un double du poète, un frère de souffrance: dénouée de toute lumière divine, l'expérience du Christ se réduit à une occasion pour méditer sa propre existence et ses douleurs ( « La front dans mes deux mains, je m'assis sur la pierre / Pensant à ce qu'avait pensé ce front divin, / Et repassant en moi, de leur source à leur fin, / Ces larmes dont le cours a creusé ma carrière »), et contribue à ébranler la foi du poète en Dieu, comme en témoigne l'un des derniers vers : «La prière en mon sein avec l'espoir est morte ». De même, Victor Hugo dans le poème qui clôt les Contemplations, À celle qui est restée en France, écrit en 1855, évoque le Gethsémani dans sa complainte pour la fille disparue : à nouveau le lieu de l'agonie du 
Christ renvoie à la souffrance de l'homme face au mal («Toujours nous arrivons à ta grotte fatale, / Gethsémani, qu'éclaire une vague lueur! / O rocher de l'étrange et funèbre sueur ! / Cave où l'esprit combat le destin! ouverture / Sur les profonds effrois de la sombre nature !»)

Dans cette série romantique, il faudrait insérer aussi Le Reniement de Saint Pierre de Baudelaire, de 1852, qui dans la troisième strophe présente un Christ priant vainement un Père qui l'a abandonné aux souffrances les plus atroces (" - Ah! Jésus, souviens-toi du Jardin des Olives! / Dans ta simplicité tu priais à genoux / Celui qui dans son ciel riait au bruit des clous / Que d'ignobles bourreaux plantaient dans tes chairs vives ») ${ }^{8}$, passage qui introduit le thème de la révolte de l'homme contre un Dieu méchant.

7 Ces lectures, et notamment celles de Vigny et de Nerval, sont souvent mises par la critique en rapport avec le roman Siebenkäs de l'allemand Jean-Paul Richter, qui les aurait inspirées: c'est Baldensperger le premier qui, en 1912, met en relief cette source allemande ${ }^{9}$, rendue célèbre en France par Mme de Staël, qui dans De l'Allemagne cite le passage du roman où le Christ fait son apparition dans un cimetière et tient aux morts qui l'implorent un discours tragique dénonçant l'absence de Dieu face à ses prières ${ }^{10}$. Bien que Mme de Staël présente le récit comme destiné à prouver que «l'athéisme ne devrait pas mettre à l'abri de la crainte des souffrances éternelles " $^{11}$, celui-ci nourrira, comme nous l'avons vu, l'imaginaire romantique dans une direction franchement antichrétienne. Et cependant il nous faut introduire une précision: si Nerval revendique explicitement sa dette envers Jean-Paul par l'épigraphe du Christ aux Oliviers, la dette de Vigny est moins évidente et moins certaine ${ }^{12}$. D'autres influences apparaissent davantage cruciales pour le Mont des Oliviers: François Germain et André Jarry, dans leur annotation de la Pléiade, signalent notamment l'importance de la lecture de Strauss, le théologien allemand auteur de la Vie de Jésus, et l'influence du bouddhisme, connu par Vigny à travers l'ouvrage de Barthélemy Saint-Hilaire, Le Bouddha et sa religion ${ }^{13}$. Ces lectures nous permettent en effet de pénétrer certains passages du poème : les inspirations venant du bouddhisme auraient inspiré les vers conclusifs qui exhortent au silence, et la lecture de Strauss peut être à l'origine du vers sur l'humanité du Christ (« devint homme », v. 27), bien que l'expression "le fils de l'Homme», employée au v. 21 et au v. 144, se trouve dans Matthieu avant d'apparaître chez Strauss, et encore que Vigny ne manque pas de noter dans son Journal un jugement perplexe face au projet de la Vie de Jésus essayant de distinguer dans le christianisme le côté historique d'avec le côté mythique («Le docteur Strauss a fait sur le Nouveau Testament le même travail que Spinoza sur l'ancien. C'est un procès instruit pesamment, en demande de nullité de divinité et de vérité historique ») ${ }^{14}$. Pour saisir la signification véritable et les origines de la réflexion sur la religion proposée dans le Mont des Oliviers, il faut en réalité élargir le réseau des références de Vigny.

Tout d'abord, l'intérêt de Vigny pour le thème de la Passion du Christ est ancien, datant des temps de sa première tragédie juvénile Roland, jamais achevée, mais qui représente une source " intra-textuelle »; un passage du Journal nous précise en effet : «Il n'y avait de supportable dans le Roland qu'un vers, sur Jésus-Christ: "Fils exilé du ciel, tu souffris au désert" $»^{15}$.

9 Ensuite, dans la strophe conclusive dite "du Silence », Vigny peut avoir été influencé par Lord Byron : l'acte de révolte radicale qui est ici préconisé rappelle l'acte byronien, même si Vigny invite au « dédain » plutôt qu'à une opposition directe ${ }^{16}$. 
10 Finalement et surtout, le passage central du poème où le Christ revendique une religion porteuse de «Certitude heureuse et $\left[\mathrm{d}^{\prime}\right]$ Espoir confiant » dévoile une autre référence fondamentale. La polémique du Christ, et de Vigny avec lui, se porte contre la souffrance que Dieu inflige durablement aux hommes, disposé tel qu'il est à sacrifier son Fils avant que celui-ci ait complété son œuvre de diffusion d'un message de paix et fraternité (« $\mathrm{Ne}$ sens-tu pas le monde et tout le genre humain / Qui souffre avec ma chair et frémit dans ta main? », vv. 37-38). La terre demeure ainsi en proie au «manteau de misère » que le Christ n'a que brièvement soulevé («N'ayant que soulevé ce manteau de misère / Qui l'entoure à grand plis, drap lugubre et fatal, / Que d'un bout tient le Doute et de l'autre le Mal », vv. 84-86). Ce que Vigny reproche à Dieu n'est ni son absence (comme le faisait Jean-Paul), ni son inexistence (nous sommes loin de la mort de Dieu de Nietzsche), mais sa méchanceté, son indifférence face aux souffrances des hommes. Or, cette douleur est évoquée à deux reprises par des mots empruntés à Voltaire : au vers 110, Vigny décrit les souffrances qui se développent «Entre la Léthargie et les Convulsions » en s'inspirant du Candide (chapitre XXX : «Martin [...] conclut que l'homme était né pour vivre dans les convulsions de l'inquiétude, ou dans la léthargie de l'ennui »); au vers 118, il décrit les esprits du mal qui triomphent "Des maux immérités, de la mort des enfants » en reprenant le Poème sur le désastre de Lisbonne de Voltaire ("Quel crime, quelle faute ont commis ces enfants »). Plusieurs critiques ont souligné l'importance de Voltaire pour Vigny, et entre autres René Pomeau, qui en dressant un bilan des querelles sur la religion de Voltaire définit le Christ aux Oliviers de Vigny comme étant tout à la fois voltairien et

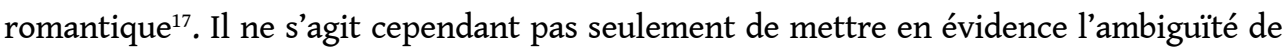
Vigny qui d'un côté conçoit le romantisme comme un antidote contre le "désenchantement" des Lumières, et de l'autre partage l'approche rationnelle à la religion qui est celle de Voltaire, fondée notamment sur le libre examen ${ }^{18}$. Vigny entretient de fait une dette profonde envers l'héritage des Lumières, qu'il est possible d'évaluer dans sa signification et dans toute son importance à partir de l'analyse d'une comparaison qui revient souvent au tournant des XVIII ${ }^{e}$ et XIX ${ }^{e}$ siècles, celle entre la mort du Christ et la mort de Socrate.

Voltaire aussi avait proposé une lecture de la Passion du Christ. C'est dans un passage du Traité de la tolérance, où la mort du Christ est décrite dans les termes suivants :

Si Jésus-Christ sembla craindre la mort, si l'angoisse qu'il ressentit fut si extrême qu'il en eut une sueur mêlée de sang, ce qui est le symptôme le plus violent et le plus rare, c'est qu'il daigna s'abaisser à toute la faiblesse du corps humain qu'il avait revêtu. Son corps tremblait, et son âme était inébranlable ; il nous apprenait que la vraie force, la vraie grandeur consistent à supporter des maux sous lesquels notre nature succombe... Il y a un extrême courage à courir la mort en la redoutant. 19

12 Cette présentation de Jésus dans une lumière presque héroïque, proposée par Voltaire dans l'un de ses moments les moins antichrétiens, est précédée par un long développement autour du parallèle entre Jésus et Socrate. Il s'agit d'une comparaison aux origines anciennes, dressée pour la première fois par Justin de Naplouse dans ses Apologies des chrétiens, et que l'on retrouve entre autres aussi chez Clément, Origène, Augustin, et jusqu'à Marc Aurèle et Lucien ${ }^{20}$. Voltaire revient dans son parallèle sur plusieurs arguments traditionnels : Socrate et Jésus sont à ses yeux similaires parce que tous les deux périssent "par la haine » (celle des sophistes et des prêtres pour le premier, des scribes, des pharisiens et des prêtres pour le second); ils vont tous les deux à l'encontre de la mort sans l'éviter; ils pardonnent à leurs ennemis, qu'ils tiennent pour « ignorants 
ou aveugles"; et montrent de respecter la loi, ne voulant pas fonder des sectes ${ }^{21}$. Nonobstant les tons de louange, ce parallèle est fonctionnel à la présentation de Jésus en moraliste, et à l'atténuation donc de la portée divine de son enseignement. La confrontation prend forme en effet dans le texte pour expliquer la mort de Jésus d'un point de vue complètement humain («sa mort, humainement parlant, a beaucoup de rapport avec celle de Socrate»); Jésus aurait en commun avec Socrate l'éthique du pardon, à laquelle aucune spécificité chrétienne n'est reconnue; tous les deux auraient pour ennemis les « insipientes ", ce qui assimile la méconnaissance du mystère divin à la pure et simple absence de savoir. Voltaire atténue davantage la nature divine du Christ lorsqu'il tient, dans la lecture de la Passion qui clôt ce passage, à reconduire la «sueur mêlée de sang " des Évangiles à un phénomène physique ${ }^{22}$; et il se borne à tirer de l'épisode évangélique non pas une leçon sur la douleur, mais une réflexion toute terrestre sur la mort en tant que menace majeure pour l'homme.

13 Si Socrate devient au cours du XVIII e siècle une sorte de "saint laïque " (pour d'Holbach aussi entre autres) ${ }^{23}$, celle-ci ne sera pas la seule lecture possible. Dans les mêmes années, Rousseau propose une lecture très différente, en opposition directe à celle de Voltaire. Voici un passage de la Profession de foi du vicaire savoyard:

Quels préjugés, quel aveuglement ne faut-il point avoir pour oser comparer le fils de Sophronisque au fils de Marie? Quelle distance de l'un à l'autre! Socrate, mourant sans douleur, sans ignominie, soutint aisément jusqu'au bout son personnage ; et si cette facile mort n'eût honoré sa vie, on douterait si Socrate, avec tout son esprit, fut autre chose qu'un sophiste. [...] La mort de Socrate, philosophant tranquillement avec ses amis, est la plus douce qu'on puisse désirer; celle de Jésus expirant dans les tourments, injurié, raillé, maudit de tout un peuple, est la plus horrible qu'on puisse craindre. [...] Oui, si la vie et la mort de Socrate sont d'un sage, la vie et la mort de Jésus sont d'un Dieu. ${ }^{24}$

La mort et la Passion du Christ sont tenues ici pour incomparables avec celles de Socrate : la souffrance et la douleur en constituent la spécificité, et elles représentent pour Rousseau une valeur essentielle dans la mesure où elles constituent la voie d'accès à un dialogue intime, immédiat, profond, avec l'humanité. C'est à partir de ce présupposé fondamental, et au sein d'une polémique contre les abstractions de la raison, que le parallèle entre Socrate et Jésus trouve donc sa place dans la Profession de foi du vicaire savoyard, se développant dans le sillage des réflexions du théologien protestant Jacob Vernet, que Rousseau avait $\mathrm{lu}^{25}$.

Quelques années plus tard, au début du XIX ${ }^{\mathrm{e}}$ siècle, la comparaison entre Socrate et le Christ devient un véritable cliché, que l'on retrouve sous la plume de maints auteurs: Bowman, qui consacre à la "Confirmatio christianorum per socratica » un chapitre bref, mais essentiel de son Christ romantique, signale parmi les auteurs qui mettent en avant cette association Mme de Staël, George Sand, Lamennais (selon qui la mort de Jésus est plus héroïque que celle de Socrate), Éliphas Lévi (selon qui Socrate ne s'adresse qu'aux intellectuels, tandis que le Christ parle à l'homme ordinaire), Victor Hugo (selon qui Socrate et Jésus sont tous les deux victimes d'une société injuste ${ }^{26}$. On peut distinguer ainsi des significations diverses de cette association, de celles à matrice apologétique, qui entendent affirmer la supériorité de la religion chrétienne, à celles à matrice syncrétiste, qui superposent et assimilent les cultes indifféremment, à celles aux présupposés plus sociaux et politiques, comme dans le cas de Hugo ${ }^{27}$. Comment se situent dans ce cadre les auteurs que l'on a vu proposer un Gethsémani de désespoir et d'angoisse face à l'absence de Dieu? 

Phédon de Platon présente les derniers instants de la vie du philosophe, et notamment le dialogue que celui-ci entretient avec ses disciples. Il s'agit d'une conversation sereine, qui se développe dans le cadre d'une nature harmonieuse, et qui présente la mort comme étant une libération et un passage heureux à une vie supérieure. "Qu'est-ce donc que mourir ? [...] / Mourir n'est pas mourir, mes amis, c'est changer!»; et Socrate de conclure son discours avec joie : «Aux dieux libérateurs, dit-il, qu'on sacrifie! / Ils m'ont guéri! - De quoi ? dit Cébès. - De la vie !... $»^{28}$. Socrate va rejoindre un Dieu nommé au singulier: aucune discontinuité n'est repérée entre les cultes grecs et la religion chrétienne.

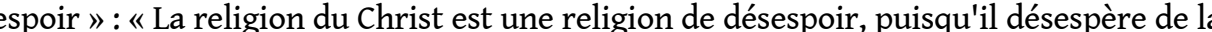
vie et n'espère que dans l'éternité $»^{30}$. Si le récit évangélique de la Passion frappe Vigny, c'est en raison de la notion de sacrifice et non pas de celle de souffrance : «L'humanité devait tomber à genoux devant cette histoire [du Christ] parce que le sacrifice est ce qu'il $\mathrm{y}$ a de plus beau au monde, et qu'un Dieu né sur la crèche et mort sur la croix dépasse les bornes des plus grands sacrifices $»^{31}$. Il reste fidèle à cette conception même lorsqu'il modifie partiellement ses positions antichrétiennes, pour récupérer la valeur sociale du christianisme. Vigny tient pour inconciliables la douleur et la divinité ( Il est un Dieu? J'ai pourtant bien souffert!»32, et il projette en 1835 la pièce Élévation, qui voudrait raconter une tentative de révolte contre le Dieu méchant du christianisme :

Dieu voit avec orgueil un jeune homme illustre sur la terre. Or ce jeune homme était très malheureux et se tua avec une épée. Lorsque son âme parut devant Dieu, Dieu lui dit: "Qu'as-tu fait ? pourquoi as-tu détruit ton corps ?" L'âme répondit : "C'est pour $t$ 'affliger et te punir. Car pourquoi m'avez-vous créé malheureux ? Et pourquoi avez-vous créé le mal de l'âme, le péché, et le mal du corps, la souffrance ? Fallait-il vous donner plus longtemps le spectacle de mes douleurs ?

Vigny est en cela solidaire avec le Voltaire de l'Épittre à Uranie s'opposant à « [...] un Dieu que je devrais haïr; / un Dieu qui nous forma pour être misérables, / qui nous donne des cœurs coupables / pour avoir droit de nous punir, / nous fit à lui-même semblables / afin de nous avilir, / et nous faire à jamais sentir / les maux les plus insupportables ${ }^{34}$; refusant avec Dieu l'Incarnation aussi («Le Fils d'un charpentier expira sur la Croix », v. 95), et essayant de substituer un Dieu bon (qui « nous juge sur nos vertus, / et non pas sur nos sacrifices ", vv. 140-141) à un Dieu chrétien («je ne reconnais point à cette indigne image / le Dieu que je dois adorer », vv. 96-97; « on te fait un tyran, en toi je cherche un 
Père ; ", v. 104). Du côté de Voltaire, se rangera aussi quelques années après Stendhal, dont le Julien Sorel refuse le Dieu de la Bible « petit despote cruel, plein de la soif de se venger », en faveur du « Dieu de Voltaire, juste, bon, infini ».

21 Sans insister davantage sur les analogies entre les attitudes de Voltaire et Vigny envers la religion (il faudrait à ce propos évoquer entre autres le refus de l'incarnation et la recherche de l'« Esprit pur " ${ }^{35}$, ainsi qu'une conception de la religion qui oscille entre la cosmogonie et la dictée morale $)^{36}$, nous nous attacherons en guise de conclusion à montrer qu'à partir des affinités et des différences dans le traitement du thème de la souffrance de l'homme et du Christ, il est possible de repérer une série de couples d'oppositions qui sont en mesure de mettre en évidence des dialogues, des dettes, des parcours au sein de l'histoire littéraire.

Tout d'abord, l'assimilation Socrate-Christ, qui s'appuie comme on l'a vu sur la dépréciation de souffrance, s'accompagne toujours d'une opposition à l'œuvre de Pascal : si les Lettres philosophiques de Voltaire ont dans les Pensées de Pascal l'une de leurs cibles polémiques principales (sont contestées notamment les Pensées qui portent sur la misère de l'homme et sur le mélange de misère et grandeur) ${ }^{37}$, Vigny aussi ne manque de critiquer sévèrement l'approche de Pascal. La réfutation de celui-ci est notamment au cœur du projet de la Deuxième consultation:

Le Docteur remarque bien que l'Évangile, l'Imitation de Jésus-Christ, le livre des Pensées de Pascal et tous les livres qui attestent le christianisme, n'ont qu'une idée, c'est le néant de toute chose humaine, et le mépris de la vie et du temps, en vue de la mort et de l'éternité. [...], mais ne la [la vie] rendez pas plus mauvaise qu'elle n'est, en vous créant des imaginations qui ne peuvent aboutir qu'à vous rendre fous. ${ }^{38}$

Pascal se serait en effet perdu, nonobstant la vigueur de son esprit, pour avoir voulu considérer la religion autrement que pour sa fonction sociale :

Plus l'esprit est vigoureux, plus il se perd dans les catacombes de l'incertitude humaine. Pascal s'y est perdu pour avoir marché plus avant que les autres. Toute religion n'a jamais été crue qu'à moitié et a eu ses athées et ses sceptiques. Mais les sages ont gardé leurs doutes dans leur cœur et ont respecté la fable sociale reçue généralement et adoptée du plus grand nombre. ${ }^{39}$

Les polémiques liées à la lecture de Pascal - dont l'œuvre selon Cassirer représente « le plus grand défi lancé à tout le XVIII ${ }^{e}$ siècle $»^{40}$ - se poursuivront en ce sens jusqu'au début $\mathrm{du} \mathrm{XX}^{\mathrm{e}}$ siècle, lorsque des auteurs tels que Barrès, Péguy et Du Bos conduiront leur bataille contre le positivisme en revenant à Pascal, et en le défendant contre ses détracteurs ${ }^{41}$.

Deuxièmement, un couple d'opposition double prend forme, dont les extrémités sont d'un côté Chateaubriand et Voltaire, et d'un autre côté Vigny et Maistre. À la polémique explicite qui oppose Chateaubriand et Voltaire, et qui marque la discontinuité entre les deux siècles, correspond en effet l'opposition entre Vigny et Maistre, qui annonce différents courants du romantisme. L'enjeu de l'opposition est dans les deux cas le même : le mystère de l'injustice, qui constitue une incarnation ultérieure du mystère de la souffrance que l'on a vu être au cœur de la réflexion sur Socrate, Jésus et leurs morts respectives. Dans le chapitre du Génie du christianisme consacré à la rédemption, Chateaubriand attaque Voltaire précisément au sujet du mal dans le monde : «Comment les larmes, les chagrins, les malheurs du juste, comment les triomphes et les succès impunis du méchant, comment, dis-je, sans une chute première, tout cela pourrait-il $s^{\prime}$ expliquer? $\gg^{42}$; et il propose une lecture conséquente du passage évangélique de la 
Passion, qu'il faudrait méditer à ses yeux non pas avec «l'esprit », mais avec le « cœur », suivant des termes proches de ceux employés par Rousseau :

Ne le demandons point à notre esprit, mais à notre cœur, nous tous faibles et coupables, comment un Dieu peut mourir. Si ce parfait modèle du bon fils, cet exemple des amis fidèles, si cette retraite au Mont des Oliviers, ce calice amer, cette sueur de sang, cette douceur d'âme, cette sublimité d'esprit, cette croix, ce voile déchiré, ce rocher fendu, ces ténèbres de la nature, si ce Dieu enfin expirant pour les hommes, ne peut ravir notre cœur, ni enflammer nos pensées, il est à craindre qu'on ne trouve jamais dans nos ouvrages, comme dans ceux du Poète, "des miracles éclatants", Speciosa miracula. ${ }^{43}$

La défense de la souffrance comme étant une composante essentielle de l'existence se prolonge sous la plume de Maistre, qui voit dans le principe de réversibilité le présupposé essentiel de l'incarnation et du sacrifice du Christ; et Vigny dans son Mont des Oliviers s'oppose précisément à ce dogme, en refusant d'une manière radicale l'approche qui est celle de Maistre ("Sur la tête de ceux qui viendront en disant : " "Il est permis pour tous de tuer l'innocent" ", vv. 61-62). Bien que la critique contre-révolutionnaire de Maistre ne soit pas complètement étrangère à l'héritage des Lumières, et malgré la polémique que l'auteur des Soirées de Saint Petersburg entretient contre Rousseau, son attitude face à la question de la souffrance décide de sa place au tournant du XVIII ${ }^{\mathrm{e}}$ et du XIX ${ }^{\mathrm{e}}$ siècle, ainsi que de la fascination qu'il ne cessera d'exercer sur bien de ses épigones antimodernes jusqu'au $\mathrm{XX}^{\mathrm{e}}$ siècle ${ }^{44}$.

Finalement, il est possible d'arrêter un troisième couple d'opposition : refuser le Dieu « absconditus » et la misère de l'homme de Pascal revient à se tourner vers la philosophie classique, stoïcienne notamment, à la recherche d'une éthique de la souffrance différente de l'éthique catholique. Dans son projet théâtral de 1843 déjà mentionné, Vigny songe à une scène païenne pour accueillir la révolte de l'âme contre Dieu; dans ses notes pour Daphné (où un rôle essentiel est par ailleurs joué par le stoïcien Basile de Césarée), Vigny conçoit un projet ayant pour titre Les Stoïciens, et visant à développer la critique contre la «faiblesse» du christianisme, que l'on a vue dénoncée aussi dans le Journal: «Le christianisme a rendu l'homme larmoyant. C'est ce que je veux faire sentir dans Julien l'Apostat $»^{45}$; «Le platonisme est un parfum qui ne peut être respiré que par les têtes les plus hautes et déjà nourries et embaumées de leurs fortes pensées. Le christianisme est un fruit à la portée de la faiblesse. La faiblesse est éternelle, et il sera éternellement bon à l'éducation toujours nécessaire des masses faibles et des Barbares sans cesse renaissants ${ }^{46}$. C'est dans le dernier vers de La mort du loup que l'on trouve énoncé clairement l'idéal de mort de Vigny, très différent de celui de la Passion évangélique : « Puis après, comme moi, Souffre et meurs sans parler $»^{47}$. Cette leçon qui vient de la sagesse païenne est pour Vigny dans un rapport de continuité directe avec l'enseignement de Voltaire : dans la conclusion de Daphné, la figure du réformateur religieux qu'est Julien l'Apostat (à qui Vigny déclare d'ailleurs s'identifier) est assimilée à celle de Voltaire ${ }^{48}$. De nouveau, on est face à une association qui connaîtra une longue existence, et qui jouira notamment d'un prestige essentiel à la fin du XIX ${ }^{\mathrm{e}}$ siècle, lorsque la critique envers la religion formulée par Renan et par Taine sur le plan philosophique ainsi que par maints poètes symbolistes sur le plan poétique, ira de pair avec un retour à la philosophie stoïcienne, élevée au rôle de nouvelle « religion laïque $»^{49}$.

28 Dans le parcours que nous avons déployé à partir de quelques lectures romantiques de la Passion du Christ, nous avons donc pu repérer des chemins, des filiations littéraires et philosophiques, qui ne valident pas la partition critique entre «Lumières » et 
" romantisme ", se déployant sans solution de continuité à la fois à travers le XVIII et le $\mathrm{XIX}^{\mathrm{e}}$ siècle. Nous avons tout d'abord pu ainsi rapprocher Vigny et Lamartine de Voltaire, pour leurs lectures de la mort du Christ et du parallèle Socrate-Christ ; et nous avons pu opposer à ce premier groupe de lectures un deuxième groupe, qui lui est alternatif et qui trouve son initiateur en Rousseau. Mais il a été aussi possible de cerner les enjeux qui décident de la diversité de ces lectures. Premièrement, la valeur et l'importance attribuées à la souffrance de l'homme, et avec elle à l'incarnation humaine de la divinité, ce Christ qui connaît toute la vulnérabilité des hommes ; ensuite, le jugement porté sur la justice et l'injustice de Dieu par rapport au mal qu'il laisse exister dans le monde, un jugement qui se révèle de fait aller de pair avec la valeur attribuée à la souffrance. Si d'un côté le refus de la souffrance, et avec elle de Dieu pour son caractère injuste, s'accompagne d'une attitude rationaliste-progressiste propre à Voltaire et à Vigny et qui sera héritée par le positivisme de la fin du $\mathrm{XIX}^{\mathrm{e}}$ siècle, d'un autre côté prend forme une tradition qui voit dans l'acceptation de la douleur et de la misère du monde la voie pour accéder à une vie humaine plus digne : il s'agit de la tradition qui va de Pascal à Rousseau et aux antimodernes du tournant du XIX ${ }^{e}$ et XX $\mathrm{XX}^{\mathrm{e}}$ siècle, parmi lesquels on peut compter Péguy, dont la lecture de la Passion insiste précisément sur la valeur positive de la faiblesse du Christ. Nous espérons non seulement avoir ainsi exploré le dialogue profond que le début du XIX ${ }^{e}$ siècle entretient avec les Lumières au sujet de l'épisode évangélique qui constitue le nœud tragique et essentiel de la religion catholique; mais aussi avoir contribué à ouvrir des pistes pour repérer, dans l'histoire littéraire, des chemins de transmission, de circulation et de survivance des réflexions et des idées.

\section{NOTES}

1. J. Fabre, Lumières et romantisme, nouvelle édition revue et augmentée, Paris, Klincksieck, 1980 [1963].

2. Sur la dispute entre Érasme et John Colet sur la signification de la prière de Jésus au Père, voir leur échange dans Opus epistolarum Des. Erasmi Roterodami, denuo recognitum et auctum per P. S. Allen, vol. I, Oxonii, in typographeo Clarendoniano, 1906, p. 245-260 : tandis que Colet croit que la douleur de Jésus découle surtout de la commisération du péché commis par les Juifs, Érasme tient la résignation douloureuse du Christ face à son supplice pour une preuve de sa nature humaine, et de sa douceur; Thomas More essaie de concilier ces deux positions dans son Jésus au Gethsémani. De tristitia Christi (cf. trad. italienne, con introduzione e note di D. Pezzini, Milano, S. Paolo, 2001).

3. A. de Vigny, Le Mont des Oliviers, dans Id., CEuvres complètes, vol. I, texte présenté, établi et annoté par F. Germain et A. Jarry, Paris, Gallimard, 1986, p. 149. Ce poème de Vigny a fait l'objet dans les années 1970 d'un vif débat interprétatif, portant sur la nature chrétienne ou antichrétienne de son contenu : cf. J. M. McGoldrick, "Vigny's Unorthodox Christ », dans Modern Language Notes, vol. 85, n. 4, mai 1970, p. 510-514 ; L. Bishop, « Jesus as Romantic Hero : Le Mont des Oliviers ", dans The French Review, Special Issue, n. 5, avril 1973, p. 41-48.

4. A. de Vigny, Euvres, op. cit., I, p. 153. 
5. G. de Nerval, Le Christ aux Oliviers, dans Id., CEuvres complètes, vol. III, édition publiée sous la direction de J. Guillaume et de Cl. Pichois, Paris, Gallimard, 1993, p. 648-651.

6. A. de Lamartine, Gethsémani ou La mort de Julia, dans Id., Euvres poétiques, édition présentée, établie et annotée par M.-F. Guyard, Paris, Gallimard, 1963, p. 561.

7. V. Hugo, À celle qui est restée en France, dans Id., CEuvres poétiques, vol. II, édition établie et annotée par P. Albouy, Paris, Gallimard, 1967, p. 831.

8. C. Baudelaire, Le Reniement de Saint Pierre (Les Fleurs du mal), dans Id., Euvres complètes, vol. I, texte établi, présenté et annoté par Cl. Pichois, Paris, Gallimard, 1975, p. 121.

9. F. Baldensperger, Alfred de Vigny, contribution à sa biographie intellectuelle, Paris, Hachette, 1912, notamment le chapitre «Le Songe de Jean-Paul dans le romantisme français », p. 159-176.

10. "J'ai parcouru les mondes, je me suis élevé au-dessus des soleils, et là aussi il n'est point de Dieu ; je suis descendu jusqu'aux dernières limites de l'univers, j'ai regardé dans l'abîme et je me suis écrié : - Père, où es-tu? mais je n'ai entendu que la pluie qui tombait goutte à goutte dans l'abîme, et l'éternelle tempête, que nul ordre ne régit, m'a seule répondu. Relevant ensuite mes regards vers la voûte des cieux, je n'y ai trouvé qu'une orbite vide, noire et sans fond. L'éternité reposait sur le chaos et le rongeait et se dévorait lentement elle-même : redoublez vos plaintes amères et déchirantes ; que des cris aigus dispersent les ombres, car c'en est fait ", citation tirée de Mme de Staël, De l'Allemagne, Paris, Flammarion, 1968, t. II, p. 55. Pour l'édition allemande, $c f$. J. P. Richter, Siebenkäs, hrsg. von Klaus Pauler, München, Edition Text + Kritik, 1991.

11. Ibid., p. 53.

12. Cf. Cl. Pichois, L'image de Jean-Paul Richter dans les lettres françaises, Paris, Corti, 1963.

13. Cf. A. de Vigny, Euvres, op. cit., I, p. 1104-1105.

14. Id., Journal d'un poète, Paris, Éditions Aujourd'hui, 1981 [réédition de l'édition Lemerre, Paris, 1885], p. 151.

15. Ibid., p. 28

16. Cf. sur ce point J.-P. Lassalle, Alfred de Vigny, Paris, Fayard, 2010, notamment le chapitre « Vigny et le silence de Dieu », p. 386-389.

17. R. Pomeau, La religion de Voltaire, Paris, Nizet, 1969.

18. $C f$. à ce sujet aussi M. Citoleux, Alfred de Vigny: persistances classiques et affinités étrangères, Paris, Champion, 1924.

19. Voltaire, Traité sur la tolérance, ed. by H. Mason and J. Renwick, dans Les Euvres complètes de Voltaire, vol. 56C, Oxford, Voltaire Foundation, 2000, p. 223.

20. Cf. Th. Deman, Socrate et Jésus, Paris, L'Artisan du livre, 1944.

21. Voltaire, Traité sur la tolérance, op. cit., p. 222-224.

22. J. Renwick signale dans son commentaire que c'est dans le sillage du Commentaire littéral de la Bible par Calmet (Dissertation sur la sueur du sang) que Voltaire propose cette explication de la «sueur de sang ».

23. D’Holbach, Le Christianisme dévoilé ou Examen des principes et des effets de la Religion chrétienne, Paris, Libraires Associés, 1767.

24. J.-J. Rousseau, Émile ou De l'éducation, Paris, Flammarion, 2009, p. 445-446.

25. Cf. la note de P.-M. Masson dans son édition de la Profession de foi du vicaire savoyard (édition critique d'après les manuscrits de Genève, Neuchâtel et Paris, avec une introduction et un commentaire historique par P.-M. Masson, Genève, Slatkine, 2011, réimpression de l'édition de Fribourg-Paris, 1914, p. 405 et sqq.), qui met en rapport le parallèle de Rousseau avec les écrits du théologien J. Vernet, notamment le Traité de la Vérité de la Religion chrétienne, Genève, Gosse, 1748-55, où, après une énumération des points en commun entre Socrate et Jésus, l'auteur conclut que Jésus a une "autorité infiniment plus respectable", "le [Socrate] surpasse de beaucoup ", pour attaquer finalement ceux qui le diminuent "jusqu'à le traiter de visionnaire et d'extravagant. Je laisse à juger s'il y eut jamais de partialité plus injuste ». 
26. F. P. Bowman, Le Christ romantique, Genève, Droz, 1973, chap. « La confirmatio christianorum per Socratica ", p. 141-150. Cf. aussi Id., Le Christ des barricades : 1789-1848, Paris, Éd. du Cerf, 1987. 27. Sur le syncrétisme romantique, $c f$. P. Moreau, «Romantisme français et syncrétisme religieux », dans Id., Âmes et thèmes romantiques, Paris, Corti, 1965, p. 147-164.

28. A. de Lamartine, Euvres poétiques, op. cit., p. 85-108.

29. A. de Vigny, Journal, op. cit., p. 302.

30. Ibid., p. 98 (1834).

31. Ibid., p. 43.

32. Id., Euvres, op. cit., I, p. 67.

33. Id., Journal, op. cit., p. 108 (1835).

34. Voltaire, Épître à Uranie, dans Les cuvres complètes de Voltaire, vol. 1B (Euvres de 1707-1722), Oxford, Voltaire Foundation, 2002, p. 463-502, vv. 24-31.

35. G.F. Bonnefoy, La pensée religieuse et morale d'Alfred de Vigny, Paris, Hachette, 1940, e J. Sungolowsky, Alfred de Vigny et le dix-huitième siècle, Paris, Nizet, 1968.

36. Cf. la pensée sur "Croyance ou religion » que Vigny note en 1843 dans son Journal, op. cit., p. $181-183$.

37. Cf. Voltaire, Lettres philosophiques, édition de F. Deloffre, Paris, Gallimard, 1986, notamment p. 160.

38. A. de Vigny, Stello dans CEuvres complètes, vol. II, texte présenté, établi et annoté par A. Bouvet, Paris, Gallimard, 1993, p. 993.

39. Id., Journal, op. cit., p. 161.

40. E. Cassirer, La Philosophie des lumières, traduit de l'allemand et présenté par Pierre Quillet, Paris, Fayard, 1966.

41. Cf. A. Compagnon, «Le funeste Pascal », dans Revue d'Histoire Littéraire de la France, 2007/2, p. 413-432.

42. F.-R. de Chateaubriand, Génie du christianisme, t. I, Paris, Garnier-Flammarion, 1966, p. 67.

43. Ibid., p. 68.

44. Cf. A. Compagnon, Les antimodernes de Joseph de Maistre à Roland Barthes, Paris, Gallimard, 2005. Au sujet de Maistre, $c f$. aussi M. Froidefont, Théologie de Joseph de Maistre, Paris, Éditions Classiques Garnier, 2010.

45. A. de Vigny, Documents sur Daphné, dans Euvres, op. cit., II, p. 985.

46. Ibid., p. 1026.

47. Ibid., I, p. 145. Sur le stoïcisme de Vigny, $c f$. M. Spanneut, Permanence du stoïcisme de Zénon à Malraux, Paris, J. Duculot, 1973, et G.F. Bonnefoy, op. cit.

48. A. de Vigny, Daphné, dans Id., Euvres, op. cit., II, p. 980 : « Ils regardèrent la statue de Julien. À ses pieds était Luther, et plus bas Voltaire qui riait ». Pour l'identification entre Vigny et Julien, cf. ibid., p. 985 : « Je ne puis vaincre la sympathie que j'ai toujours eue pour Julien l'Apostat. Si la métempsycose existe, j'ai été cet homme. C'est l'homme dont le rôle, la vie, le caractère m'eussent le mieux convenu dans l'histoire ».

49. Cf. notre article «Le Christ au Gethsémani de Charles Péguy entre Pascal et Renan », dans Revue d'Histoire Littéraire de la France, 2013/2, p. 341-356. 
INDEX

Mots-clés : Lumières, romantisme, Vigny (Alfred de), Lamartine (Alphonse de), Voltaire, Nerval (Gérard de), Passion du Christ, Hugo (Victor) 